\title{
Levantamento Fitossociológico de Plantas Daninhas na Cultura do Girassol ${ }^{1}$
}

\author{
Phytosociological Survey of Weeds in Sunflower Crop
}

ADEGAS, F.S. ${ }^{2}$, OLIVEIRA, M.F. ${ }^{2}$, VIEIRA, O.V. ${ }^{3}$, PRETE, C.E.C. ${ }^{4}$, GAZZIERO, D.L.P. ${ }^{2}$ e VOLL, E. ${ }^{2}$

\begin{abstract}
RESUMO - O levantamento fitossociológico da comunidade de plantas daninhas na cultura do girassol foi realizado em duas épocas distintas: no desenvolvimento inicial da cultura (entre 20 e 40 dias após a semeadura) e na pré-colheita do girassol. As espécies de plantas daninhas foram identificadas e quantificadas pelo método do quadrado inventário $(1,0 \times 1,0 \mathrm{~m})$, com amostragem de $12 \mathrm{~m}^{2}$ por área. Os levantamentos foram realizados em 54 propriedades de seis municipios da região do cerrado e em 38 propriedades de oito municipios da região dos pampas, que são as duas principais regiões produtoras brasileiras. Foram registrados a frequência, a frequência relativa, a densidade, a densidade relativa, a abundância, a abundância relativa, o indice de importância relativa e o índice de similaridade. No total, foram identificadas 60 espécies de plantas daninhas, sendo 17 presentes em ambas as regiões. Asteraceae e Poaceae foram as duas principais familias, entre as 16 encontradas. As principais espécies presentes no cerrado foram Euphorbia heterophylla, Chamaesyce hirta, Ageratum conyzoides, Commelina benghalensis, Zea mays e Bidens sp. As principais espécies presentes no Rio Grande do Sul foram Bidens sp., Raphanus raphanistrum, Lolium multiflorum, Gnaphalium spicatum, Sonchus oleraceus, Euphorbia heterophylla, Sida rhombifolia, Digitaria sp. e Ipomea sp. A densidade das plantas daninhas foi maior na fase de pré-colheita do que no desenvolvimento inicial da cultura, em ambas as regiões, sendo de 30,84 plantas $\mathrm{m}^{-2}$ e 23,58 plantas $\mathrm{m}^{-2}$, respectivamente, para o cerrado, e de 23,19 plantas $\mathrm{m}^{-2}$ e 21,41 plantas $\mathrm{m}^{-2}$, para o Rio Grande do Sul. O indice de similaridade dentro das regiões foi de 0,91 para os levantamentos do cerrado e de 0,79 para os do Rio Grande do Sul. Entretanto, entre as regiões, os indices ficaram abaixo de 0,5, mostrando similaridade mediana entre a flora daninha do cerrado e a do Rio Grande do Sul, na cultura do girassol, nas duas épocas estudadas.
\end{abstract}

Palavras-chave: competição, fitossociologia, Helianthus annuus, infestação.

\begin{abstract}
A phytosociological survey of the weed communities present in sunflower crop was carried out during two distinct crop stages: early development and pre-harvest. Weed species were identified and quantified according to the inventory square method $(1.0 \times 1.0 \mathrm{~m})$, using $12 \mathrm{~m}^{2}$ of sampling per area surveyed. At each stage, the survey was carried out on 54 farms of six counties of the "cerrado" (savanna region, Central Brazil) and 38 farms of eight counties of the "Pampas" (region in the State of Rio Grande do Sul, the Southernmost state in Brazil), which are the two main Brazilian sunflower production regions. Frequency, relative frequency, density, relative density, abundance, relative abundance, relative importance index, and similarity index were computed. A total of 60 weed species were identified, 17 of which were present in both regions. Asteraceae and Poaceae were the two main families among the 16 found. The main species present in the "cerrado" were Euphorbia heterophylla, Chamaesyce hirta, Ageratum conyzoides, Commelina benghalensis, Zea mays and Bidens sp. The most abundant species present in the State of Rio Grande do Sul were Bidens sp., Raphanus raphanistrum, Lolium multiflorum, Gnaphalium spicatum, Sonchus oleraceus, Euphorbia heterophylla, Sida rhombifolia, Digitaria sp. and Ipomea sp. Weed density was higher at pre-harvest than at the early stage of the crop, in both
\end{abstract}

Recebido para publicação em 6.10.2009 e na forma revisada em 12.11.2010.

2 Dr., Pesquisador da Embrapa Soja, Caixa Postal 231, 86001-970 Londrina-PR; ${ }^{3}$ Dr., Pesquisador da Embrapa Trigo, Rod. BR 285 , Km 291, 99001-970 Passo Fundo-RS; ${ }^{4}$ Dr., Professor do Dep. de Fitotecnia, Universidade Estadual de Londrina - UEL, Caixa Postal 6001, 86051-990 Londrina-PR. 
regions: 30.84 plants $m^{-2}$ and 23.58 plants $m^{-2}$, respectively, for the "cerrado" region and 23.19 plants $m^{-2}$ FORMATAR and 21.41 plants $m^{-2}$, respectively, for the "Pampas" region. The similarity index within each region was 0.91 forthe "cerrado" and 0.79 forthe "Pampas". Nevertheless, the region indices remained below 0.5 , showing median similarity between the weed flora affecting sunflower crop at the "cerrado" and that at the "Pampas" during the two growth stages studied.

Keywords: competition, phytosociology, Helianthus annuus, infestation

\section{INTRODUÇÃO}

O estabelecimento de uma comunidade de plantas daninhas depende das condições locais, como tipo de solo, clima, práticas culturais utilizadas, banco de sementes, etc. Por isso, pode ocorrer de forma variada nas diversas regiões produtoras de uma determinada cultura, como verificado por Saturnino \& Rocha (1993) e Laca-Buendia et al. (1995) em levantamentos realizados em diferentes áreas de cultivo da soja.

O manejo de plantas daninhas é uma das principais práticas adotadas no sistema de produção do girassol. A interferência dessas plantas pode resultar em perda de produtividade, menor qualidade do produto colhido ou aumento do custo de produção da cultura. A base para a formulação de uma eficiente proposta de controle é o conhecimento da flora daninha que ocorre nas áreas de cultivo.

Nos últimos anos, os Estados de Goiás, Mato Grosso do Sul e Rio Grande do Sul têm representado mais de $80 \%$ da área cultivada e da produção de girassol no Brasil (CONAB, 2009). Portanto, para se obter um diagnóstico adequado sobre a situação da infestação de plantas daninhas nessa cultura, são essas as principais regiões nas quais devem-se realizar os levantamentos.

Na região do cerrado, o cultivo do girassol ocorre logo após a colheita da cultura de verão, que normalmente é a soja ou o milho. Na região dos pampas, o girassol é cultivado no período de inverno/primavera, antecedendo a cultura de verão, sendo essas, portanto, as épocas mais propícias para a realização de levantamentos da flora daninha na cultura.

Em um levantamento florístico, além da identificação das espécies infestantes, há também a necessidade da análise quantitativa dessas espécies, que se denomina de estudo ou método fitossociológico (Braun-Blanquet, 1979), o qual fornece dados específicos das espécies presentes, como frequência, densidade e abundância, e também a sua relação com a população total de infestantes. Assim, o método fitossociológico é uma ferramenta que permite fazer várias inferências sobre a flora daninha em questão (Erasmo et al., 2004).

No Brasil, estudos fitossociológicos foram realizados para diversas culturas, como o arroz irrigado (Erasmo et al., 2004), o cafeeiro (LacaBuendia \& Brandão, 1994), a cana-de-açúcar (Oliveira \& Freitas, 2008; Kuva et al., 2008), o guaraná (Albertino et al., 2004), o milho (Duarte \& Deuber, 1999; Macedo et al., 2003), as pastagens (Carvalho \& Pitelli, 1992; Mascarenhas et al., 1999; Lara et al., 2003; Tuffi Santos et al., 2004) e a soja (Saturnino \& Rocha, 1993; Laca-Buendia et al., 1995).

Geralmente, os estudos de fitossociologia de comunidades infestantes são realizados em uma fase determinada da cultura, normalmente próximo à colheita, quando teoricamente a infestação daninha é máxima - período em que foram realizados os levantamentos por Bedmar (1983) e por Brighenti et al. (2003), na cultura do girassol. No entanto, o período crítico de prevenção de interferência (PCPI) das plantas daninhas com as culturas agrícolas, que é quando a interferência promove perdas para a cultura (Pitelli, 1985), nem sempre ocorre na fase final da cultura. Pelo contrário, quase sempre ocorre na fase inicial dela, como é o caso do girassol, em que o PCPI foi estipulado entre 28 e 42 dias após a emergência em trabalhos realizados nos Estados Unidos (Johnson, 1971) e na Índia (Ghosh et al., 1979); entre 20 e 30 dias na Argentina (Bochicchio \& Arregui, 1974); e entre 21 e 30 dias, após a emergência, no 
Brasil (Brighenti et al., 2004). Isso mostra a importância de se proceder aos levantamentos da flora daninha nessa fase da cultura.

Objetivou-se com este trabalho identificar e quantificar as principais plantas daninhas presentes nas fases de desenvolvimento inicial e de pré-colheita na cultura do girassol, por meio de levantamento fitossociológico realizado nas principais regiões produtoras da oleaginosa no Brasil

\section{MATERIAL E MÉTODOS}

Os levantamentos foram realizados em duas épocas distintas de desenvolvimento do girassol: entre 20 e 40 dias após a emergência da cultura, denominada de época de desenvolvimento inicial (DSI), e na pré-colheita (PRÉ). Na região do cerrado, nos Estados de Goiás e Mato Grosso do Sul, eles foram realizados em março (DSI) e em maio (PRÉ); e na região dos pampas, no Rio Grande do Sul, em setembro (DSI) e em dezembro (PRÉ). O número de propriedades e a área amostrada por município foram, respectivamente, 18 e $216 \mathrm{~m}^{2} \mathrm{em}$ Chapadão do Céu (GO), 6 e $72 \mathrm{~m}^{2}$ em Jataí (GO), 14 e $168 \mathrm{~m}^{2}$ em Montividiu (GO), 3 e $36 \mathrm{~m}^{2}$ em Rio Verde (GO), 3 e $36 \mathrm{~m}^{2}$ em Iporá (GO), 10 e $120 \mathrm{~m}^{2}$ em Chapadão do Sul (MS), 8 e $96 \mathrm{~m}^{2}$ em Horizontina (RS), 2 e $24 \mathrm{~m}^{2}$ em Ibirubá (RS), 4 e $48 \mathrm{~m}^{2}$ em Ijuí (RS), 7 e $84 \mathrm{~m}^{2}$ em Jóia (RS), 3 e $36 \mathrm{~m}^{2}$ em Saldanha Marinho (RS), 5 e $60 \mathrm{~m}^{2}$ em Santa Rosa (RS), 6 e $72 \mathrm{~m}^{2}$ em Três de Maio (RS) e 3 e $36 \mathrm{~m}^{2}$ em XV de Novembro (RS).

As plantas daninhas foram identificadas e quantificadas pelo método do quadrado inventário (Braun-Blanquet, 1979), em que foi utilizado um quadrado de $1,0 \times 1,0 \mathrm{~m}$ para demarcar aleatoriamente 12 áreas amostradas no interior das lavouras. Além da quantificação das espécies e do total dos indivíduos por área amostrada, foram ainda calculados os seguintes parâmetros fitossociológicos propostos por Mueller-Dombois \& Ellenberg (1974):

Frequência $=\frac{\mathrm{n}^{\mathrm{o}} \text { de quadrados onde a espécie foi encontrada }}{\mathrm{n}^{\mathrm{o}} \text { total de quadrados }}$

Frequência relativa $=\frac{\text { frequência da espécie } \mathrm{x} 100}{\text { frequência total das espécies }}$

Densidade $=\frac{\mathrm{n}^{\mathrm{o}} \text { total de indivíduos da espécie }}{\mathrm{n}^{\mathrm{o}} \text { total de quadrados }}$

Densidade relativa $=\frac{\text { densidade da espécie } \mathrm{x} 100}{\text { densidade total das espécies }}$

Abundância $=\frac{\mathrm{n}^{\mathrm{o}} \text { total de indivíduos da espécie }}{\mathrm{n}^{\mathrm{o}} \text { total de quadrados onde a espécie foi encontrada }}$

Abundância relativa $=\frac{\text { abundância da espécie } \times 100}{\text { abundância total das espécies }}$

Índice de importância relativa $=$ frequência relativa + densidade relativa + abundância relativa 
Também foi calculado o coeficiente de similaridade, segundo a fórmula proposta por Sorensen (1972):

Coeficiente de similaridade $=$

$2 \times \mathrm{n}^{\mathrm{O}}$ de espécies comuns aos dois habitats

$\mathrm{n}^{\mathrm{o}}$ de espécies do habitat $\mathrm{A}+\mathrm{n}^{\mathrm{o}}$ de espécies do habitat $\mathrm{B}$

\section{RESULTADOS E DISCUSSÃO}

Foram identificadas 41 espécies, agrupadas em 13 famílias, nos levantamentos realizados na região do cerrado (Tabela 1). As principais famílias presentes foram Asteraceae, Poaceae, Euphorbiaceae e Fabaceae, com 11, 10, 4 e 3 espécies cada, respectivamente.

Na região dos pampas foram identificadas 15 famílias, número maior que nos levantamentos realizados nos municípios da região do cerrado, mas com menor diversidade de espécies, sendo identificadas 37 (Tabela 2). As duas principais famílias também foram a Asteraceae, com 10 espécies, e a Poaceae, com sete espécies, seguidas pela família Brassicaceae, com cinco.

Asteraceae e Poaceae realmente são duas das principais familias de plantas daninhas existentes no Brasil, pois, além de estarem presentes em áreas tradicionais de produção de grãos, como as de girassol, também aparecem com grande importância em outros sistemas diferenciados de produção, como o da cana-de-açúcar (Oliveira \& Freitas, 2008), de explorações de várzeas (Tuffi Santos et al., 2004) e até em áreas de gramados (Maciel et al., 2008).

No total, foram identificadas 60 diferentes espécies de plantas daninhas, sendo 17 nas duas regiões estudadas. Espécies da família Cyperaceae foram encontradas apenas na região do cerrado, enquanto espécies das famílias Caryophyllaceae, Polygonaceae e Sapindaceae só foram identificadas nos pampas.

Na região do cerrado, as principais espécies observadas no levantamento realizado no início de desenvolvimento da cultura do girassol (Tabela 3) foram Euphorbia heterophylla, Chamaesyce hirta, Ageratum conyzoides e Commelina benghalensis. Algumas espécies, como Richardia brasiliensis e
Tridax procumbens, tiveram concentração de indivíduos em áreas específicas - característico das infestações destas espécies (Kissmann \& Groth, 1999) -, o que resultou em indice de frequência baixa, mas de abundância alta. Brighenti et al. (2003) encontraram resultados semelhantes para estas duas espécies também em levantamento fitossociológico da cultura do girassol no cerrado, com Tridax procumbens tendo frequência de 0,058 e abundância de 4,588 plantas $\mathrm{m}^{-2}$, enquanto Richardia brasiliensis mostrou frequência de 0,007 e abundância de 2,250 plantas $\mathrm{m}^{-2}$.

Das 20 principais espécies, com Ir acima de 3\% (tabela 3), 17 são dicotiledôneas, o que dificulta o controle químico das plantas daninhas no girassol, pois, no Brasil, só há dois herbicidas registrados para a cultura: o alachlor e a trifluralina (Brasil, 2009), ambos com baixa eficiência no controle de magnoliopsidas na cultura do girassol (Castro et al., 1997).

Ainda na região do cerrado, comparando o levantamento de pré-colheita (Tabela 4) com o do desenvolvimento inicial (Tabela 3), observa-se que o número de espécies encontradas na cultura do girassol aumentou de 36 para 39, assim como a quantidade de indivíduos, de 15.283 para 19.987 , o que resultou em aumento de 23,58 plantas $\mathrm{m}^{-2}$ para 30,84 plantas $\mathrm{m}^{-2}$. Isso ocorreu pela germinação e emergência tardia das plantas daninhas, em relação ao desenvolvimento do girassol, especialmente algumas magnoliopsidas, que é comum acontecer na região do cerrado, como mostrou o trabalho de Teixeira et al. (2009), os quais estudaram a competição de plantas daninhas com a cultura do feijão e verificaram maior importância da infestação de dicotiledôneas na época das secas (outono/inverno) do que no cultivo das águas (primavera/verão).

Por ordem de importância, as principais espécies encontradas na pré-colheita foram Ageratum conyzoides, Chamaesyce hirta, 
Bidens sp., Euphorbia heterophylla e Commelina benghalensis. Os resultados são muito seme1hantes aos obtidos por Brighenti et al. (2003), que catalogaram 42 espécies, em levantamentos realizados na pré-colheita da cultura do girassol, nos mesmos municipios de Chapadão do Céu, Chapadão do Sul, Jataí e Montividiu, coincidindo, inclusive, com as seis principais espécies encontradas neste trabalho.

Tabela 1 - Relação de plantas daninhas, distribuídas por família e espécie, presentes em lavouras de girassol na região do cerrado, nos municípios de Chapadão do Céu, Iporá, Jataí, Montividiu e Rio Verde-GO e Chapadão do Sul-MS. 2008

\begin{tabular}{|c|c|c|}
\hline Família & Nome científico & Nome comum \\
\hline \multirow{2}{*}{ Amaranthaceae } & Alternanthera tenella & apaga-fogo, periquito, alecrim, manjericão \\
\hline & Amaranthus sp. & caruru, bredo \\
\hline \multirow{11}{*}{ Asteraceae } & Acanthospermum australe & carrapicho-rasteiro, carrapichinho \\
\hline & Acanthospermum hispidum & carrapicho-de-carneiro, espinho-de-carneiro \\
\hline & Ageratum conyzoides & mentrasto, erva-de-são-joão, picão-roxo \\
\hline & Bidens sp. & picão, picão-preto, pico-pico, piolho-de-padre \\
\hline & Conyza bonariensis & buva, voadeira, rabo-de-foguete \\
\hline & Eclipta alba & erva-de-botão, agrião-do-brejo, erva-lanceta, lanceta \\
\hline & Emilia sonchifolia & falsa-serralha, pincel, bela-emília, serralhinha, brocha \\
\hline & Galinsoga parviflora & picão-branco, fazendeiro, botão-de-ouro \\
\hline & Guizotia abyssinica & níger voluntário \\
\hline & Melampodium perfoliatum & estrelinha, botão-de-cachorro \\
\hline & Tridax procumbens & erva-de-touro \\
\hline Brassicaceae & Raphanus raphanistrum & nabo, nabiça, rabanete-de-cavalo \\
\hline Commelinaceae & Commelina benghalensis & trapoeraba, rabo-de-cachorro, andaca, maria-mole \\
\hline Convolvulaceae & Ipomoea sp. & corda-de-viola, corriola, campainha \\
\hline Cyperaceae & Cyperus sp. & tiririca, junquinho \\
\hline \multirow{4}{*}{ Euphorbiaceae } & Chamaesyce hirta & erva-de-santa-luzia, burra-leiteira, erva-de-sangue \\
\hline & Chamaesyce hyssopifolia & erva-andorinha, burra-leiteira \\
\hline & Euphorbia heterophylla & amendoim-bravo, leiteiro, leiteira, flor-de-poetas \\
\hline & Phyllanthus tenellus & quebra-pedra, erva-pombinha, arrebenta-pedra \\
\hline \multirow{3}{*}{ Fabaceae } & Desmodium tortuosum & desmódio, carrapicho-beiço-de-boi, pega-pega \\
\hline & Glycine $\max$ & soja voluntária \\
\hline & Senna obtusifolia & fedegoso, mata-pasto-liso, fedegoso-branco \\
\hline \multirow{2}{*}{ Lamiaceae } & Leonotis nepetifolia & cordão-de-frade, cordão-de-são-francisco, corindiba \\
\hline & Leucas martinicensis & hortelã, mentinha, falsa-menta \\
\hline Malvaceae & Sida rhombifolia & guaxuma, mata-pasto, vassourinha \\
\hline \multirow{10}{*}{ Poaceae } & Brachiaria decumbens & capim-braquiária, braquiária \\
\hline & Brachiaria plantaginea & capim-marmelada, papuã \\
\hline & Cenchrus echinatus & capim-carrapicho, capim-amoroso, timbete \\
\hline & Digitaria insularis & capim-amargoso, capim-açu, capim-flecha, capim-pororó \\
\hline & Digitaria $\mathrm{sp}$. & capim-colchão, capim-de-roça, milhã \\
\hline & Eleusine indica & capim-pé-de-galinha, pé-de-galinha, capim-de-pomar \\
\hline & Panicum maximum & capim-colonião, capim-coloninho, capim-guiné \\
\hline & Rhynchelytrum repens & capim-favorito, favorito, capim-natal \\
\hline & Sorghum bicolor & sorgo voluntário \\
\hline & Zea mays & milho voluntário \\
\hline \multirow{2}{*}{ Rubiaceae } & Richardia brasiliensis & poaia, poaia-branca, poaia-do-campo \\
\hline & Spermacoce latifolia & erva-quente, poaia-do-campo, erva-de-lagarto \\
\hline \multirow{2}{*}{ Solanaceae } & Nicandra physaloides & joá-de-capote, quintilho, balão \\
\hline & Solanum americanum & maria-pretinha, erva-moura, caraxixá, pimenta-de-galinha \\
\hline
\end{tabular}


Tabela 2 - Relação de plantas daninhas, distribuídas por família e espécie, identificadas em lavouras de girassol na região dos pampas (RS), nos municípios de Horizontina, Ibirubá, Ijuí, Joia, Saldanha Marinho, Santa Rosa, Três de Maio e XV de Novembro. 2008

\begin{tabular}{|c|c|c|}
\hline Família & Nome científico & Nome comum \\
\hline Amaranthaceae & Amaranthus sp. & caruru, bredo \\
\hline \multirow{10}{*}{ Asteraceae } & Acanthospermum hispidum & carrapicho-de-carneiro, espinho-de-carneiro \\
\hline & Ageratum conyzoides & mentrasto, erva-de-são-joão, picão-roxo \\
\hline & Artemisia verlotorum & losna, losna-brava, artemija, artemísia, absinto \\
\hline & Bidens sp. & picão, picão-preto, pico-pico, piolho-de-padre \\
\hline & Conyza bonariensis & buva, voadeira, rabo-de-foguete \\
\hline & Galinsoga parviflora & picão-branco, fazendeiro, botão-de-ouro \\
\hline & Gnaphalium spicatum & macela, macela-branca, meloso, macio, macelinha \\
\hline & Senecio brasiliensis & maria-mole, berneira, flor-das-almas, cravo-do-campo \\
\hline & Sonchus oleraceus & serralha, serralheira, chicória-brava \\
\hline & Vernonia sp. & assapeixe, assa-peixe \\
\hline \multirow{5}{*}{ Brassicaceae } & Brassica rapa & mostarda, couve-nabeira, nabo-branco \\
\hline & Brassica napus & canola voluntária \\
\hline & Cardamine bonariensis & agrião-bravo, agriãozinho \\
\hline & Coronopus didymus & mentruz, mastruço, mentrusto \\
\hline & Raphanus raphanistrum & nabo, nabiça, rabanete-de-cavalo \\
\hline \multirow{2}{*}{ Caryophyllaceae } & Spergula arvensis & espérgula, gorga, pega-pinto \\
\hline & Stellaria media & erva-de-passarinho, esparguta, esperguta \\
\hline Commelinaceae & Commelina benghalensis & trapoeraba, rabo-de-cachorro, andaca, maria-mole \\
\hline Convolvulaceae & Ipomoea $\mathrm{sp}$. & corda-de-viola, corriola, campainha \\
\hline Euphorbiaceae & Euphorbia heterophylla & amendoim-bravo, leiteiro, leiteira, flor-de-poetas \\
\hline Fabaceae & Vicia sativa & ervilhaca, vica, avica \\
\hline \multirow{2}{*}{ Lamiaceae } & Leonorus sibiricus & rubim, erva-macaé, chá-de-frade, erva-do-santo-fillho \\
\hline & Leonotis nepetifolia & cordão-de-frade, cordão-de-são-francisco, corindiba \\
\hline Malvaceae & Sida rhombifolia & guaxuma, mata-pasto, vassourinha \\
\hline \multirow{7}{*}{ Poaceae } & Avena sp. & aveia voluntária, aveia-brava, aveia selvagem \\
\hline & Brachiaria plantaginea & capim-marmelada, papuã \\
\hline & Cenchrus echinatus & capim-carrapicho, capim-amoroso, timbete \\
\hline & Chloris sp. & capim-de-rhodes, capim branco, pé-de-galinha \\
\hline & Digitaria sp. & capim-colchão, capim-de-roça, milhã \\
\hline & Lolium multiflorum & azevém, joio, azevém-italiano \\
\hline & Zea mays & milho voluntário \\
\hline Polygonaceae & Polygonum convolvulus & cipó-de-veado, cipó-de-veado-de-inverno, enredadeira \\
\hline Rubiaceae & Richardia brasiliensis & poaia, poaia-branca, poaia-do-campo \\
\hline Sapindaceae & Cardiospermum halicacabum & balãozinho, saco-de-padre, olho-de-pomba, chumbinho \\
\hline \multirow{2}{*}{ Solanaceae } & Solanum americanum & maria-pretinha, erva-moura, caraxixá, pimenta-de-galinha \\
\hline & Solanum sisymbrifolium & joá-bravo, joá, juá, arrebenta-cavalo, mata-cavalo \\
\hline
\end{tabular}

Na região dos pampas, as principais espécies presentes no levantamento realizado no desenvolvimento inicial da cultura do girassol (Tabela 5) foram Bidens sp., Raphanus raphanistrum, Lolium multiflorum, Gnaphalium spicatum e Sonchus oleraceus. Devido à época do levantamento, início de setembro, nove das 12 principais espécies encontradas foram típicas de outono/inverno para a região Sul
(Lorenzi, 2000). Essas espécies são frequentes nas culturas de inverno dessa região, como comprovaram Agostinetto et al. (2008) em estudo de competição de plantas daninhas com a cultura do trigo no Rio Grande do Sul, onde encontraram como principais espécies infestantes Raphanus raphanistru e Lolium multiflorum, com 228 e 24 plantas $\mathrm{m}^{-2}$, respectivamente. 
Tabela 3 - Número de presença em quadrados (NQ), número de indivíduos (NI), frequência (F), frequência relativa (Fr), densidade (D), densidade relativa (Dr), abundância (A), abundância relativa (Ar) e índice de importância relativa (Ir) das espécies daninhas presentes nas lavouras de girassol em desenvolvimento inicial, na região do cerrado, nos municípios de Chapadão do Céu, Iporá, Jataí, Montividiu e Rio Verde-GO e Chapadão do Sul-MS. 2008

\begin{tabular}{|c|c|c|c|c|c|c|c|c|c|}
\hline Espécie & NQ & $\mathrm{NI}$ & $\mathrm{F}$ & Fr $(\%)$ & $\mathrm{D}\left(\mathrm{pl} . \mathrm{m}^{-2}\right)$ & $\operatorname{Dr}(\%)$ & $\mathrm{A}$ & $\operatorname{Ar}(\%)$ & $\operatorname{Ir}(\%)$ \\
\hline Euphorbia heterophylla & 185 & 2.266 & 0,285 & 9,209 & 3,497 & 14,827 & 12,249 & 6,451 & 30,487 \\
\hline Chamaesyce hirta & 234 & 1.965 & 0,361 & 11,649 & 3,032 & 12,857 & 8,397 & 4,423 & 28,929 \\
\hline Ageratum conyzoides & 237 & 1.908 & 0,366 & 11,798 & 2,944 & 12,484 & 8,051 & 4,240 & 28,522 \\
\hline Commelina benghalensis & 204 & 2.001 & 0,315 & 10,155 & 3,088 & 13,093 & 9,809 & 5,166 & 28,414 \\
\hline Zea mays & 280 & 1.486 & 0,432 & 13,939 & 2,293 & 9,723 & 5,307 & 2,795 & 26,457 \\
\hline Bidens sp. & 232 & 1.518 & 0,358 & 11,549 & 2,343 & 9,933 & 6,543 & 3,446 & 24,928 \\
\hline Digitaria $\mathrm{sp.}$ & 92 & 1.452 & 0,142 & 4,580 & 2,241 & 9,501 & 15,783 & 8,312 & 22,393 \\
\hline Glycine $\max$ & 122 & 901 & 0,188 & 6,073 & 1,390 & 5,895 & 7,385 & 3,889 & 15,858 \\
\hline Ipomoea $\mathrm{sp}$. & 106 & 422 & 0,164 & 5,277 & 0,651 & 2,761 & 3,981 & 2,097 & 10,135 \\
\hline Richardia brasiliensis & 22 & 169 & 0,034 & 1,095 & 0,261 & 1,106 & 7,682 & 4,046 & 6,247 \\
\hline Phyllanthus tenellus & 27 & 184 & 0,042 & 1,344 & 0,284 & 1,204 & 6,815 & 3,589 & 6,137 \\
\hline Alternanthera tenella & 23 & 148 & 0,035 & 1,145 & 0,228 & 0,968 & 6,435 & 3,389 & 5,502 \\
\hline Sida rhombifolia & 13 & 84 & 0,020 & 0,647 & 0,130 & 0,550 & 6,462 & 3,403 & 4,600 \\
\hline Guizotia abyssinica & 16 & 83 & 0,025 & 0,796 & 0,128 & 0,543 & 5,188 & 2,732 & 4,072 \\
\hline Leonotis nepetifolia & 5 & 36 & 0,008 & 0,249 & 0,056 & 0,236 & 7,200 & 3,792 & 4,276 \\
\hline Amaranthus sp. & 28 & 103 & 0,043 & 1,394 & 0,159 & 0,674 & 3,679 & 1,937 & 4,005 \\
\hline Tridax procumbens & 2 & 15 & 0,003 & 0,100 & 0,023 & 0,098 & 7,500 & 3,950 & 4,148 \\
\hline Spermacoce latifolia & 3 & 19 & 0,005 & 0,149 & 0,029 & 0,124 & 6,333 & 3,335 & 3,609 \\
\hline Solanum americanum & 6 & 34 & 0,009 & 0,299 & 0,052 & 0,222 & 5,667 & 2,984 & 3,506 \\
\hline Acanthospermum australe & 17 & 62 & 0,026 & 0,846 & 0,096 & 0,406 & 3,647 & 1,921 & 3,173 \\
\hline Eclipta alba & 12 & 45 & 0,019 & 0,597 & 0,069 & 0,294 & 3,750 & 1,975 & 2,867 \\
\hline Rhynchelytrum repens & 13 & 46 & 0,020 & 0,647 & 0,071 & 0,301 & 3,538 & 1,864 & 2,812 \\
\hline Leucas martinicensis & 22 & 58 & 0,034 & 1,095 & 0,090 & 0,380 & 2,636 & 1,388 & 2,863 \\
\hline Cyperus sp. & 11 & 39 & 0,017 & 0,548 & 0,060 & 0,255 & 3,545 & 1,867 & 2,670 \\
\hline Digitaria insularis & 12 & 38 & 0,019 & 0,597 & 0,059 & 0,249 & 3,167 & 1,668 & 2,514 \\
\hline Eleusine indica & 16 & 44 & 0,025 & 0,796 & 0,068 & 0,288 & 2,750 & 1,448 & 2,533 \\
\hline Braquiaria plantaginea & 5 & 20 & 0,008 & 0,249 & 0,031 & 0,131 & 4,000 & 2,107 & 2,486 \\
\hline Sorghum bicolor & 19 & 35 & 0,029 & 0,946 & 0,054 & 0,229 & 1,842 & 0,970 & 2,145 \\
\hline Brachiaria decumbens & 6 & 17 & 0,009 & 0,299 & 0,026 & 0,111 & 2,833 & 1,492 & 1,902 \\
\hline Conyza bonariensis & 7 & 18 & 0,011 & 0,348 & 0,028 & 0,118 & 2,571 & 1,354 & 1,820 \\
\hline Nicandra physaloides & 4 & 12 & 0,006 & 0,199 & 0,019 & 0,079 & 3,000 & 1,580 & 1,858 \\
\hline Emilia sonchifolia & 2 & 6 & 0,003 & 0,100 & 0,009 & 0,039 & 3,000 & 1,580 & 1,719 \\
\hline Cenchrus echinatus & 15 & 23 & 0,023 & 0,747 & 0,035 & 0,150 & 1,533 & 0,808 & 1,705 \\
\hline Galinsoga parviflora & 1 & 3 & 0,002 & 0,050 & 0,005 & 0,020 & 3,000 & 1,580 & 1,649 \\
\hline Raphanus raphanistrum & 5 & 12 & 0,008 & 0,249 & 0,019 & 0,079 & 2,400 & 1,264 & 1,591 \\
\hline Senna obtusifolia & 5 & 11 & 0,008 & 0,249 & 0,017 & 0,072 & 2,200 & 1,159 & 1,480 \\
\hline Total & $648^{1 /}$ & 15.283 & 3,100 & 100,00 & 23,585 & 100,00 & 189,878 & 100,00 & - \\
\hline
\end{tabular}

1/ Número total de quadrados, sem repetição.

Semelhantemente ao ocorrido na região do cerrado, das 20 principais espécies encontradas na região dos pampas, agora com indice de importância relativa acima de 5\% (Tabela 5), 17 são magnolipsidas, o que demonstra a dificuldade do manejo de infestantes no girassol também nesta região do País, pois não há herbicidas eficientes registrados para o controle dessa classe de plantas daninhas na cultura.
Os resultados da pré-colheita (Tabela 6) mostram que também houve germinação de espécies infestantes após a primeira época de levantamento na região dos pampas, que é uma situação normal e que resultou no aumento do número de espécies encontradas, de 27 para 34, só que em proporção menor em comparação ao aumento do número total de indivíduos, de 9.763 para 10.756 , com aumento de 21,41 plantas $\mathrm{m}^{-2}$ para 23,19 plantas $\mathrm{m}^{-2}$. 
Essa infestação é muito próxima da observada por Moraes et al. (2009), que foi de 28,6 plantas $\mathrm{m}^{-2}$ para a mesma época do ano, em estudo de controle de plantas daninhas na cultura do milho no Rio Grande do Sul.
Diferentemente do levantamento inicial, as cinco principais espécies encontradas na précolheita - Bidens sp., Euphorbia heterophylla, Sida rhombifolia, Digitaria sp. e Ipomea sp. são tipicamente de primavera/verão

Tabela 4 - Número de presença em quadrados (NQ), número de indivíduos (NI), frequência (F), frequência relativa (Fr), densidade (D), densidade relativa (Dr), abundância (A), abundância relativa (Ar) e índice de importância relativa (Ir) das espécies daninhas presentes em lavouras de girassol na pré-colheita, na região do cerrado, nos municípios de Chapadão do Céu, Iporá, Jataí, Montividiu e Rio Verde-GO e Chapadão do Sul-MS. 2008

\begin{tabular}{|c|c|c|c|c|c|c|c|c|c|}
\hline Espécie & NQ & NI & $\mathrm{F}$ & $\operatorname{Fr}(\%)$ & $\mathrm{D}\left(\mathrm{pl} . \mathrm{m}^{-2}\right)$ & $\operatorname{Dr}(\%)$ & $\mathrm{A}$ & $\operatorname{Ar}(\%)$ & $\operatorname{Ir}(\%)$ \\
\hline Ageratum conyzoides & 339 & 5.065 & 0,523 & 14,224 & 7,816 & 25,342 & 14,941 & 7,741 & 47,307 \\
\hline Chamaesyce hirta & 385 & 3.836 & 0,594 & 16,154 & 5,920 & 19,193 & 9,964 & 5,162 & 40,509 \\
\hline Bidens sp. & 291 & 2.993 & 0,449 & 12,210 & 4,619 & 14,975 & 10,285 & 5,329 & 32,514 \\
\hline Euphorbia heterophylla & 146 & 2.035 & 0,225 & 6,126 & 3,140 & 10,182 & 13,938 & 7,222 & 23,529 \\
\hline Commelina benghalensis & 175 & 930 & 0,270 & 7,343 & 1,435 & 4,653 & 5,314 & 2,753 & 14,749 \\
\hline Cenchrus echinatus & 142 & 905 & 0,219 & 5,958 & 1,397 & 4,528 & 6,373 & 3,302 & 13,788 \\
\hline Alternanthera tenella & 84 & 664 & 0,130 & 3,524 & 1,025 & 3,322 & 7,905 & 4,096 & 10,942 \\
\hline Acanthospermum australe & 38 & 441 & 0,059 & 1,594 & 0,681 & 2,206 & 11,605 & 6,013 & 9,814 \\
\hline Rhynchelytrum repens & 28 & 265 & 0,043 & 1,175 & 0,409 & 1,326 & 9,464 & 4,904 & 7,404 \\
\hline Sida rhombifolia & 82 & 341 & 0,127 & 3,441 & 0,526 & 1,706 & 4,159 & 2,155 & 7,301 \\
\hline Phyllanthus tenellus & 64 & 326 & 0,099 & 2,685 & 0,503 & 1,631 & 5,094 & 2,639 & 6,956 \\
\hline Glycine max & 36 & 273 & 0,056 & 1,510 & 0,421 & 1,366 & 7,583 & 3,929 & 6,805 \\
\hline Ipomoea sp. & 78 & 254 & 0,120 & 3,273 & 0,392 & 1,271 & 3,256 & 1,687 & 6,231 \\
\hline Digitaria sp. & 76 & 230 & 0,117 & 3,189 & 0,355 & 1,151 & 3,026 & 1,568 & 5,908 \\
\hline Tridax procumbens & 36 & 172 & 0,056 & 1,510 & 0,265 & 0,861 & 4,778 & 2,475 & 4,847 \\
\hline Solanum americanum & 26 & 139 & 0,040 & 1,091 & 0,215 & 0,695 & 5,346 & 2,770 & 4,556 \\
\hline Conyza bonariensis & 46 & 144 & 0,071 & 1,930 & 0,222 & 0,720 & 3,130 & 1,622 & 4,272 \\
\hline Zea mays & 42 & 139 & 0,065 & 1,762 & 0,215 & 0,695 & 3,310 & 1,715 & 4,172 \\
\hline Leonotis nepetifolia & 40 & 134 & 0,062 & 1,678 & 0,207 & 0,670 & 3,350 & 1,736 & 4,084 \\
\hline Digitaria insularis & 40 & 129 & 0,062 & 1,678 & 0,199 & 0,645 & 3,225 & 1,671 & 3,995 \\
\hline Spermacoce latifolia & 9 & 51 & 0,014 & 0,378 & 0,079 & 0,255 & 5,667 & 2,936 & 3,569 \\
\hline Leucas martinicensis & 32 & 91 & 0,049 & 1,343 & 0,140 & 0,455 & 2,844 & 1,473 & 3,271 \\
\hline Guizotia abyssinica & 8 & 43 & 0,012 & 0,336 & 0,066 & 0,215 & 5,375 & 2,785 & 3,336 \\
\hline Cyperus sp. & 15 & 62 & 0,023 & 0,629 & 0,096 & 0,310 & 4,133 & 2,142 & 3,081 \\
\hline Eleusine indica & 14 & 53 & 0,022 & 0,587 & 0,082 & 0,265 & 3,786 & 1,961 & 2,814 \\
\hline Amaranthus sp. & 18 & 48 & 0,028 & 0,755 & 0,074 & 0,240 & 2,667 & 1,382 & 2,377 \\
\hline Desmodium tortuosum & 3 & 13 & 0,005 & 0,126 & 0,020 & 0,065 & 4,333 & 2,245 & 2,436 \\
\hline Panicum maximum & 11 & 34 & 0,017 & 0,462 & 0,052 & 0,170 & 3,091 & 1,601 & 2,233 \\
\hline Brachiaria decumbens & 19 & 45 & 0,029 & 0,797 & 0,069 & 0,225 & 2,368 & 1,227 & 2,249 \\
\hline Emilia sonchifolia & 14 & 34 & 0,022 & 0,587 & 0,052 & 0,170 & 2,429 & 1,258 & 2,016 \\
\hline Raphanus raphanistrum & 8 & 24 & 0,012 & 0,336 & 0,037 & 0,120 & 3,000 & 1,554 & 2,010 \\
\hline Brachiaria plantaginea & 11 & 23 & 0,017 & 0,462 & 0,035 & 0,115 & 2,091 & 1,083 & 1,660 \\
\hline Nicandra physaloides & 13 & 25 & 0,020 & 0,545 & 0,039 & 0,125 & 1,923 & 0,996 & 1,667 \\
\hline Acanthospermum hispidum & 1 & 3 & 0,002 & 0,042 & 0,005 & 0,015 & 3,000 & 1,554 & 1,611 \\
\hline Melampodium perfoliatum & 1 & 3 & 0,002 & 0,042 & 0,005 & 0,015 & 3,000 & 1,554 & 1,611 \\
\hline Sorghum bicolor & 2 & 5 & 0,003 & 0,084 & 0,008 & 0,025 & 2,500 & 1,295 & 1,404 \\
\hline Senna obtusifolia & 4 & 9 & 0,006 & 0,168 & 0,014 & 0,045 & 2,250 & 1,166 & 1,379 \\
\hline Chamaesyce hyssopifolia & 2 & 3 & 0,003 & 0,084 & 0,005 & 0,015 & 1,500 & 0,777 & 0,876 \\
\hline Richardia brasilienses & 3 & 3 & 0,005 & 0,126 & 0,005 & 0,015 & 1,000 & 0,518 & 0,659 \\
\hline Total & $648^{1 / 1}$ & 19.987 & 3,676 & 100,00 & 30,844 & 100,00 & 193,004 & 100,00 & - \\
\hline
\end{tabular}

1/ Número total de quadrados, sem repetição. 
(Lorenzi, 2000). Ruedell (1995) listou as principais plantas daninhas, tanto de outono/ inverno como de primavera/verão, presentes em lavouras de grãos no Rio Grande do Sul, as quais são semelhantes às apresentadas neste trabalho, com a diferença da inclusão de Brachiaria plantaginea e Cenchrus echinatus como espécies de incidência alta e de Sonchus oleraceus como de incidência baixa; para esta espécie, o autor afirma que a semeadura direta pode proporcionar o crescimento da sua população, o que, aparentemente, ocorreu.

$O$ coeficiente de similaridade varia de 0 a 1 , sendo máximo quando todas as espécies são comuns às duas áreas e mínimo quando não há espécies comuns. A Tabela 7 mostra que houve menor semelhança de espécies entre as lavouras da região do cerrado e da região dos pampas. O coeficiente de similaridade das espécies daninhas das lavouras do cerrado no início do desenvolvimento do girassol, em comparação com as lavouras dos pampas no início do desenvolvimento e na pré-colheita, foi de 0,44 e 0,48, respectivamente.

Na pré-colheita no cerrado e em ambas as épocas nos pampas, a similaridade foi ainda menor, com coeficiente de 0,36 para a avaliação inicial e de 0,46 para a avaliação de pré-colheita. Além das características peculiares dos solos e dos sistemas de exploração utilizados, a principal razão para esse resultado é a época de cultivo diferenciada dos dois ambientes, com o aparecimento de espécies típicas de determinadas estações do ano, como de primavera/verão, no cerrado, e de outono/ inverno, no Rio Grande do Sul.

Tabela 5 - Número de presença em quadrados (NQ), número de indivíduos (NI), frequência (F), frequência relativa (Fr), densidade (D), densidade relativa (Dr), abundância (A), abundância relativa (Ar) e índice de importância relativa (Ir) das espécies daninhas presentes em lavouras de girassol em desenvolvimento inicial, na região dos pampas (RS), nos municípios de Horizontina, Ibirubá, Ijuí, Joia, Saldanha Marinho, Santa Rosa, Três de Maio e XV de Novembro. 2008

\begin{tabular}{|c|c|c|c|c|c|c|c|c|c|}
\hline Espécie & NQ & NI & $\mathrm{F}$ & $\operatorname{Fr}(\%)$ & $\mathrm{D}\left(\mathrm{pl} \cdot \mathrm{m}^{-2}\right)$ & $\operatorname{Dr}(\%)$ & $\mathrm{A}$ & $\operatorname{Ar}(\%)$ & $\operatorname{Ir}(\%)$ \\
\hline Bidens sp. & 264 & 3.078 & 0,579 & 13,700 & 6,750 & 31,527 & 11,659 & 11,954 & 57,181 \\
\hline Raphanus raphanistrum & 137 & 1.838 & 0,300 & 7,109 & 4,031 & 18,826 & 13,416 & 13,755 & 39,691 \\
\hline Lolium multiflorum & 246 & 1.244 & 0,539 & 12,766 & 2,728 & 12,742 & 5,057 & 5,185 & 30,692 \\
\hline Gnaphalium spicatum & 199 & 639 & 0,436 & 10,327 & 1,401 & 6,545 & 3,211 & 3,292 & 20,164 \\
\hline Sonchus oleraceus & 150 & 316 & 0,329 & 7,784 & 0,693 & 3,237 & 2,107 & 2,160 & 13,181 \\
\hline Digitaria sp. & 40 & 231 & 0,088 & 2,076 & 0,507 & 2,366 & 5,775 & 5,921 & 10,363 \\
\hline Brassica napus & 27 & 175 & 0,059 & 1,401 & 0,384 & 1,792 & 6,481 & 6,645 & 9,839 \\
\hline Sida rhombifolia & 57 & 243 & 0,125 & 2,958 & 0,533 & 2,489 & 4,263 & 4,371 & 9,818 \\
\hline Polygonum convolvulus & 109 & 211 & 0,239 & 5,656 & 0,463 & 2,161 & 1,936 & 1,985 & 9,802 \\
\hline Brassica rapa & 38 & 193 & 0,083 & 1,972 & 0,423 & 1,977 & 5,079 & 5,207 & 9,156 \\
\hline Avena $\mathrm{sp}$. & 25 & 147 & 0,055 & 1,297 & 0,322 & 1,506 & 5,880 & 6,029 & 8,832 \\
\hline Coronopus didymus & 83 & 178 & 0,182 & 4,307 & 0,390 & 1,823 & 2,145 & 2,199 & 8,329 \\
\hline Euphorbia heterophylla & 79 & 174 & 0,173 & 4,100 & 0,382 & 1,782 & 2,203 & 2,258 & 8,140 \\
\hline Ageratum conyzoides & 72 & 168 & 0,158 & 3,736 & 0,368 & 1,721 & 2,333 & 2,392 & 7,849 \\
\hline Cardamine bonariensis & 42 & 155 & 0,092 & 2,179 & 0,340 & 1,588 & 3,690 & 3,784 & 7,551 \\
\hline Amaranthus sp. & 43 & 128 & 0,094 & 2,231 & 0,281 & 1,311 & 2,977 & 3,052 & 6,594 \\
\hline Richardia brasiliensis & 59 & 123 & 0,129 & 3,062 & 0,270 & 1,260 & 2,085 & 2,137 & 6,459 \\
\hline Brachiaria plantaginea & 63 & 108 & 0,138 & 3,269 & 0,237 & 1,106 & 1,714 & 1,758 & 6,133 \\
\hline Solanum sisymbrifolium & 48 & 112 & 0,105 & 2,491 & 0,246 & 1,147 & 2,333 & 2,392 & 6,030 \\
\hline Vicia sativa & 40 & 105 & 0,088 & 2,076 & 0,230 & 1,075 & 2,625 & 2,691 & 5,843 \\
\hline Cardiospermum halicacabum & 25 & 70 & 0,055 & 1,297 & 0,154 & 0,717 & 2,800 & 2,871 & 4,885 \\
\hline Galinsoga parviflora & 26 & 65 & 0,057 & 1,349 & 0,143 & 0,666 & 2,500 & 2,563 & 4,578 \\
\hline Conyza bonariensis & 35 & 42 & 0,077 & 1,816 & 0,092 & 0,430 & 1,200 & 1,230 & 3,477 \\
\hline Zea mays & 10 & 9 & 0,022 & 0,519 & 0,020 & 0,092 & 0,900 & 0,923 & 1,534 \\
\hline Ipomoea sp. & 6 & 7 & 0,013 & 0,311 & 0,015 & 0,072 & 1,167 & 1,196 & 1,579 \\
\hline Senecio brasiliensis & 3 & 3 & 0,007 & 0,156 & 0,007 & 0,031 & 1,000 & 1,025 & 1,212 \\
\hline Leonurus sibricus & 1 & 1 & 0,002 & 0,052 & 0,002 & 0,010 & 1,000 & 1,025 & 1,087 \\
\hline $\begin{array}{r}\text { Total } \\
\end{array}$ & $456^{-1}$ & 9.763 & 4,226 & 100,00 & 21,410 & 100,00 & 97,536 & 100,00 & - \\
\hline
\end{tabular}

1/ Número total de quadrados, sem repetição. 
Tabela 6 - Número de presença em quadrados (NQ), número de indivíduos (NI), frequência (F), frequência relativa (Fr), densidade (D), densidade relativa (Dr), abundância (A), abundância relativa (Ar) e índice de importância relativa (Ir) das espécies daninhas presentes em lavouras de girassol na pré-colheita, na região dos pampas (RS), nos municípios de Horizontina, Ibirubá, Ijuí, Joia, Saldanha Marinho, Santa Rosa, Três de Maio e XV de Novembro. 2008

\begin{tabular}{|c|c|c|c|c|c|c|c|c|c|}
\hline Espécie & NQ & NI & $\mathrm{F}$ & $\operatorname{Fr}(\%)$ & $\mathrm{D}\left(\mathrm{pl} . \mathrm{m}^{-2}\right)$ & $\operatorname{Dr}(\%)$ & $\mathrm{A}$ & $\operatorname{Ar}(\%)$ & $\operatorname{Ir}(\%)$ \\
\hline Bidens sp. & 304 & 2.654 & 0,667 & 17,334 & 5,820 & 25,095 & 8,730 & 6,712 & 49,14 \\
\hline Euphorbia heterophylla & 171 & 2.586 & 0,375 & 9,750 & 5,671 & 24,452 & 15,123 & 11,627 & 45,83 \\
\hline Sida rhombifolia & 113 & 945 & 0,248 & 6,443 & 2,072 & 8,935 & 8,363 & 6,430 & 21,81 \\
\hline Digitaria $\mathrm{sp}$. & 147 & 872 & 0,322 & 8,382 & 1,912 & 8,245 & 5,932 & 4,561 & 21,19 \\
\hline Ipomoea $\mathrm{sp}$ & 137 & 692 & 0,300 & 7,812 & 1,518 & 6,543 & 5,051 & 3,883 & 18,24 \\
\hline Gnaphalium spicatum & 100 & 415 & 0,219 & 5,702 & 0,910 & 3,924 & 4,150 & 3,191 & 12,82 \\
\hline Brachiaria plantaginea & 78 & 260 & 0,171 & 4,448 & 0,570 & 2,458 & 3,333 & 2,563 & 9,47 \\
\hline Sonchus oleraceus & 89 & 229 & 0,195 & 5,075 & 0,502 & 2,165 & 2,573 & 1,978 & 9,22 \\
\hline Richardia brasiliensis & 64 & 253 & 0,140 & 3,649 & 0,555 & 2,392 & 3,953 & 3,039 & 9,08 \\
\hline Amaranthus sp. & 80 & 217 & 0,175 & 4,562 & 0,476 & 2,052 & 2,713 & 2,085 & 8,70 \\
\hline Conyza bonariensis & 56 & 228 & 0,123 & 3,193 & 0,500 & 2,156 & 4,071 & 3,130 & 8,48 \\
\hline Cardiospermum halicacabum & 69 & 211 & 0,151 & 3,934 & 0,463 & 1,995 & 3,058 & 2,351 & 8,28 \\
\hline Solanum sisymbrifolium & 42 & 197 & 0,092 & 2,395 & 0,432 & 1,863 & 4,690 & 3,606 & 7,86 \\
\hline Solanum americanum & 64 & 166 & 0,140 & 3,649 & 0,364 & 1,570 & 2,594 & 1,994 & 7,21 \\
\hline Coronopus didymus & 9 & 58 & 0,020 & 0,513 & 0,127 & 0,548 & 6,444 & 4,955 & 6,02 \\
\hline Lolium multiflorum & 31 & 95 & 0,068 & 1,768 & 0,208 & 0,898 & 3,065 & 2,356 & 5,02 \\
\hline Acanthospermum hispidum & 20 & 64 & 0,044 & 1,140 & 0,140 & 0,605 & 3,200 & 2,460 & 4,21 \\
\hline Raphanus raphanistrum & 30 & 71 & 0,066 & 1,711 & 0,156 & 0,671 & 2,367 & 1,820 & 4,20 \\
\hline Cloris sp. & 13 & 46 & 0,029 & 0,741 & 0,101 & 0,435 & 3,538 & 2,720 & 3,90 \\
\hline Cardamine bonariensis & 11 & 40 & 0,024 & 0,627 & 0,088 & 0,378 & 3,636 & 2,796 & 3,80 \\
\hline Ageratum conyzoides & 29 & 59 & 0,064 & 1,654 & 0,129 & 0,558 & 2,034 & 1,564 & 3,78 \\
\hline Zea mays & 16 & 47 & 0,035 & 0,912 & 0,103 & 0,444 & 2,938 & 2,258 & 3,62 \\
\hline Commelina benghalensis & 4 & 14 & 0,009 & 0,228 & 0,031 & 0,132 & 3,500 & 2,691 & 3,05 \\
\hline Spergula arvensis & 5 & 16 & 0,011 & 0,285 & 0,035 & 0,151 & 3,200 & 2,460 & 2,90 \\
\hline Senecio brasiliensis & 20 & 36 & 0,044 & 1,140 & 0,079 & 0,340 & 1,800 & 1,384 & 2,86 \\
\hline Leonotis nepetifolia & 9 & 22 & 0,020 & 0,513 & 0,048 & 0,208 & 2,444 & 1,879 & 2,60 \\
\hline Polygonum convolvulus & 15 & 27 & 0,033 & 0,855 & 0,059 & 0,255 & 1,800 & 1,384 & 2,49 \\
\hline Cenchrus echinatus & 1 & 3 & 0,002 & 0,057 & 0,007 & 0,028 & 3,000 & 2,307 & 2,39 \\
\hline Artemisia verlotorum & 3 & 8 & 0,007 & 0,171 & 0,018 & 0,076 & 2,667 & 2,050 & 2,30 \\
\hline Brassica rapa & 2 & 5 & 0,004 & 0,114 & 0,011 & 0,047 & 2,500 & 1,922 & 2,08 \\
\hline Galinsoga parviflora & 4 & 9 & 0,009 & 0,228 & 0,020 & 0,085 & 2,250 & 1,730 & 2,04 \\
\hline Vernonia sp. & 9 & 16 & 0,020 & 0,513 & 0,035 & 0,151 & 1,778 & 1,367 & 2,03 \\
\hline Stellaria media & 7 & 11 & 0,015 & 0,399 & 0,024 & 0,104 & 1,571 & 1,208 & 1,71 \\
\hline Vicia sativa & 2 & 4 & 0,004 & 0,114 & 0,009 & 0,038 & 2,000 & 1,538 & 1,69 \\
\hline Total & $456^{1 /}$ & 10.576 & 3,846 & 100,00 & 23,193 & 100,00 & 130,067 & 100,00 & - \\
\hline
\end{tabular}

1/ Número total de quadrados, sem repetição.

Tabela 7 - Coeficiente de similaridade dos levantamentos fitossociológicos realizados em municípios da região do cerrado (Goiás e Mato Grosso do Sul) e da região dos pampas (RS), no desenvolvimento inicial (DSI) e na pré-colheita (PRÉ) das lavouras de girassol. 2008

\begin{tabular}{|l|c|c|c|}
\hline \multicolumn{1}{|c|}{ Local } & Cerrado -PRÉ & RS -DSI & RS -PRÉ \\
\hline Cerrado - DSI & 0,91 & 0,44 & 0,48 \\
\hline Cerrado - PRÉ & - & 0,36 & 0,46 \\
\hline RS - DSI & - & - & 0,79 \\
\hline
\end{tabular}

Em relação à análise dentro das regiões, houve maior semelhança entre as espécies encontradas nos levantamentos realizados no início do desenvolvimento do girassol e na précolheita, com coeficiente de similaridade de 0,91 para as lavouras do cerrado e de 0,79 para as dos pampas. Isso demonstra que as espécies presentes no desenvolvimento inicial da cultura do girassol foram semelhantes àquelas observadas na época da colheita.

No entanto, as diferenças encontradas entre os parâmetros fitossociológicos das espécies, entre as épocas avaliadas, podem ser relevantes para o planejamento do manejo das 
plantas daninhas. Com isso, evidencia-se a importância de conhecer as espécies daninhas e suas populações durante todo o ciclo da cultura, especialmente no período crítico de prevenção da interferência.

\section{LITERATURA CITADA}

AGOSTINETTO, D. et al. Período crítico de competição de plantas daninhas com a cultura do trigo. Planta Daninha, v. 26, n. 3 , p. $271-278,2008$

ALBERTINO, S. M. F. et al. Composição florística das plantas daninhas na cultura de guaraná (Paullinia cupuana), no estado do Amazonas. Planta Daninha, v. 22, n. 3, p. 351-358, 2004.

BEDMAR, F. Relevamiento de malezas en cultivo de girasol en el centro sudeste de la provincia de Buenos Aires. In: REUNIÓN ARGENTINA SOBRE LA MALEZA Y SU CONTROL, 10., 1983, Buenos Aires. Actas... Buenos Aires: ASAM, 1983. v. 11, n. 4, p. 200-208.

BOCHICCHIO, J.; ARREGUI, C. Determinacion del período de competência de malezas mediante labores em el cultivo de girasol (Helianthus annuus L.). In: REUNION NACIONAL DE GIRASOL, 2., 1974, Buenos Aires, Actas... Buenos Aires: Instituto Agroindustrial de Oleaginosas, 1974. p. 117-220.

BRAUN-BLANQUET, J. Fitossociologia: bases para el estudio de las comunidades vegetales. Madri: H. Blume, 1979. $820 \mathrm{p}$.

BRIGHENTI, A. M. et al.. Cadastramento fitossociológico de plantas daninhas na cultura do girassol. Pesq. Agropec. Bras., v. 38 , n. 5 , p. $651-657,2003$.

BRIGHENTI, A. M. et al. Período de interferência de plantas daninhas na cultura do girassol. Planta Daninha, v. 22, n. 2 , p. 251-257, 2004.

CARVALHO, S. L.; PITELLI, R. A. Levantamento e análise fitossociológica das principais espécies de plantas daninhas de pastagens da região de Selvíria (MS). Planta Daninha, v. 10, n. $1 / 2$, p. $25-32,1992$

CASTRO, C. et al. A cultura do girassol. Londrina: Embrapa-CNPSo, 1997. 36 p. (Circular Técnica, 13).

COMPANHIA NACIONAL DE ABASTECIMENTO CONAB. Acompanhamento de safra brasileira: grãos, décimo levantamento, julho/2009. Brasília: Conab, 2009. 39 p

DUARTE, A. P.; DEUBER, R. Levantamento de plantas infestantes em lavouras de milho "safrinha" no estado de São Paulo. Planta Daninha, v. 17, n. 2, p. 297-307, 1999.
ERASMO, E. A. L.; PINHEIRO, L. L. A.; COSTA, N. V. Levantamento fitossociológico das comunidades de plantas infestantes em áreas de produção de arroz irrigado cultivado sob diferentes sistemas de manejo. Planta Daninha, v. 22, n. 2, p. 195-201, 2004.

GHOSH, G. S.; SINGH, S.; FHOSH, A. K. Response of sunflower to weed control. India Pesticides, v. 13, n. 3, p. $44-46,1979$

JOHNSON, B. J. Effect of weed competition on sunflower. Weed Sci., v. 19, n. 4, p. 378-380, 1971.

KISSMANN, K. G.; GROTH, D. Plantas infestantes e nocivas. 2.ed. São Paulo: BASF, 1999. Tomo II. 978 p.

KUVA, M. A. et al. Padrões de infestação de comunidades de plantas daninhas no agroecosssistema de cana-crua.

Planta Daninha, v. 26, n. 3, p. 549-557, 2008.

LACA-BUENDIA, J. P.; BRANDÃO, M. Cadastramento e análise quantitativa das plantas daninhas ocorrentes em cafezais localizados em áreas anteriormente ocupadas pela formação Cerrado no Triângulo Mineiro e Alto Paranaíba. Daphne, v. 4, n. 4, p. 71-76, 1994.

LACA-BUENDIA, J. P. et al. Cadastramento fitossociológico de plantas daninhas na pré-colheita da cultura da soja (Glycine $\max ($ L.) Merril) no Triângulo Mineiro e Alto Paranaíba, Minas Gerais, Brasil. Daphne, v. 5, n. 3, p. 84-96, 1995.

LARA, J. F. R.; MACEDO, J. F.; BRANDÃO, M. Plantas daninhas em pastagens de várzeas no estado de Minas Gerais. Planta Daninha, v. 21, n. 1, p. 11-20, 2003

LORENZI, H. Plantas daninhas do Brasil: terrestres, aquáticas, parasitas e tóxicas. 3.ed. Nova Odessa: Instituto Plantarum, 2000. $608 \mathrm{p}$.

MACEDO, J. F.; BRANDÃO, M.; LARA, J. F. R. Plantas daninhas na pós-colheita de milho nas várzeas do rio São Francisco, em Minas Gerais. Planta Daninha, v. 21, n. 2, p. 239-248, 2003.

MACIEL, C. D. G. et al. Composição florística da comunidade infestante em gramados de Paspalum notatum no município de Assis, SP. Planta Daninha, v. 26, n. 1, p. $57-64,2008$

BRASIL. Ministério da Agricultura. Agrofit- consulta de produtos formulados. Disponível em: $<\mathrm{http}: / /$ www.agricultura.gov.br/agrofit_cons $>$. Acesso em: 10 jul 2009.

MASCARENHAS, R. E. B. et al. Plantas daninhas de uma pastagem cultivada de baixa produtividade no Nordeste Paraense. Planta Daninha, v. 17, n. 3, p. 399-418, 1999. 
MORAES, P. V. D. et al. Manejo de plantas de cobertura no controle de plantas daninhas na cultura do milho. Planta Daninha, v. 27, n. 2, p. 289-296, 2009.

MUELLER-DOMBOIS, D.; ELLEMBERG, H. A. Aims and methods of vegetation ecology. New York: John Wiley, 1974. $574 \mathrm{p}$.

OLIVEIRA, A. R.; FREITAS, S. P. Levantamento fitossociológico de plantas daninhas em áreas de produção de cana-de-açúcar. Planta Daninha, v. 26, n. 1, p. 33-46, 2008

PITELLI, R. A. Interferências de plantas daninhas em culturas agrícolas. Informe Agropecuário, n. 129, p. 16-27, 1985.

RUEDELL, J. Plantio direto na Região de Cruz Alta. Cruz Alta: FUNDACEP-FECOTRIGO, 1995. 134 p.
SATURNINO, H. M.; ROCHA, B. V. Levantamento e análise quantitativa de plantas daninhas ocorrentes no final do ciclo da soja (Glycine max (L.) Merril), em Felixlândia-MG, 1979. Daphne, v. 3, n. 3, p. 46-51, 1993.

SORENSEN, T. A. Method of stablishing groups of equal amplitude in plant society based on similarity of species content. In: ODUM, E. P. Ecologia. 3.ed. México: Interamericana, 1972. p. 341-405.

TEIXEIRA, I. R. et al. Competição entre feijoeiros e plantas daninhas em função do tipo de crescimento dos cultivares.

Planta Daninha, v. 27, n. 2, p. 235-240, 2009.

TUFFI SANTOS, L. D. et al. Levantamento fitossociológico em pastagens degradadas sob condições de várzea.

Planta Daninha, v. 22, n. 3, p. 343-349, 2004. 